\title{
ON THE ITERATION OF RATIONAL FUNCTIONS*
}

\author{
BY
}

\section{J. F. RITT}

\section{INTRODUCTION}

Notes on the iteration of rational functions have been published in the C o m p tes Rendus during the last few years by Julia, Fatou, Lattés, and myself. $\dagger$ Many of the results announced were obtained independently by more than one writer, the priority in such cases seeming to belong to Julia. I wish to present here that small part of my work which has not been covered by the other writers, postponing until after the appearance of their memoirs the publication of such of my other results as may still be of interest. $\neq$

The $p$ th iterate of the analytic function $f(x)$ will be denoted by $f_{p}(x)$. If $f(c)=c$, the point $c$ will be called a fixed point of $f(x)$. Let the Taylor development of $f(x)$ at the fixed point $c$ be

$$
f(x)=c+a_{1}(x-c)+a_{2}(x-c)^{2}+\cdots+a_{n}(x-c)^{n}+\cdots .
$$

The point $c$ will be called a point of attraction or point of repulsion according as $\left|a_{1}\right|<1$ or $\left|a_{1}\right|>1 . \S$ I have not had occasion thus far to study the case where $\left|a_{1}\right|=1$. If $\alpha_{1}=f_{p}\left(\alpha_{2}\right)$, where $p$ is some positive integer, $\alpha_{1}$ is called a consequent of $\alpha_{2}$, and $\alpha_{2}$ an antecedent of $\alpha_{1}$. If $f_{p}\left(\alpha_{1}\right)=f_{p}\left(\alpha_{2}\right)$ for some positive integer $p$, I shall say that $\alpha_{1}$ and $\alpha_{2}$ are associates of each other. If

$$
F(x)=\phi^{-1} f \phi(x),
$$

$f(x)$ will be said to be transformed into $F(x)$ by means of $\phi(x)$.

I have made use, in my work, of a theorem which was first found by L.

* Presented to the Society, December 27, 1917, and February 23, 1918.

† Julia, December 31, 1917, January 14, 1918, January 28, 1918; Fatou, May 14, 1917, December 17, 1917, February 4, 1918; Lattés, January 7, 1918, January 28, 1918, March 25, 1918; Ritt, March 4, 1918.

\$ While this paper was in the hands of the editors of the $\mathrm{Tr}$ a $\mathrm{ns}$ a $\mathrm{ct}$ i o $\mathrm{n} \mathbf{s}$, papers appeared by Julia, Journal de Mathématiques, 1918 (published one year late), and by Fatou, Bulletin de la Société Mathématique de France, 1919. Fatou's paper contains some of the details of $\S$ III of the present paper, but asfar as I can see, these results were first stated in my note in the $\mathrm{Com}$ p t e s $\mathrm{R}$ e $\mathrm{ndus}$.

$\S$ These terms were introduced by me in my note in the Comptes Rendus, and have since been used by all other writers on this subject. 
Boettcher,* who published it in Russian. To make the present paper easier to read, I have devoted $\S$ II to a very brief proof of Boettcher's theorem.

In § III I discuss the set of associates of a point.

In § IV I determine the conditions under which two polynomials can have an iterate in common.

\section{BOETTCHER'S THEOREM}

Let $f(x)$ have, for $x=0$, the Taylor development

$$
f(x)=a_{m} x^{m}+a_{m+1} x^{m+1}+\cdots+a_{m+i} x^{m+i}+\cdots,
$$

where $m>1$. Within a sufficiently small circle with center at the origin, $f_{p}(x)$ approaches zero uniformly as $p$ increases indefinitely. We consider a circle of this type, in which $f(x)$ has no zero other than $x=0$. Within this circle, every iterate $f_{p}(x),(p=1,2, \cdots, n, \cdots)$, exists, and vanishes only for $x=0$, at which point it has a zero of order $m^{p}$. The $m^{p}$ th roots of $f(x)$ will be uniform within this circle, with simple zeros at $x=0$. The first coefficient in the development of $f_{p}(x)$ is $a_{m}^{\left(m^{p}-1\right) /(m-1)}$. We can thus select a sequence of functions

$$
[f(x)]^{1 / m}, \quad\left[f_{2}(x)\right]^{1 / m^{2}}, \quad \cdots, \quad\left[f_{p}(x)\right]^{1 / m^{p}}, \quad \cdots,
$$

such that the coefficients of $x$ in the terms of the sequence approach any chosen $(m-1)$ th root of $a_{m}$. We may now state

BoEtTCHER's THEOREM. In the circle described abore, the functions in the sequence (1) converge uniformly to an analytic function $\phi(x)$, which satisfies the functional equation

$$
\phi f(x)=[\phi(x)]^{m} .
$$

We suppose first that $a_{m}=1$; the general case will be shown later to depend on this special one. We may assume then that the coefficients of $x$ in the terms of (1) are all unity.

The functions of the sequence (1) have a common upper bound for their moduli, and hence also a common majorant, in the circle under consideration. We may thus write, since as $p$ increases, $f_{p}(x)$ approaches zero uniformly with respect to $x$,

$$
f_{p+q}(x)=f_{q}\left[f_{p}(x)\right]=\left[f_{p}(x)\right]^{m^{q}}\left[1+\epsilon_{p, q}(x)\right],
$$

where $\epsilon_{p, q}(x)$ tends toward zero as $p$ increases, uniformly with respect to $x$ and $q$. Consequently,

$$
\left[f_{p+q}(x)\right]^{1 / m^{p+q}}=\left[f_{p}(x)\right]^{1 / m^{p}}\left[1+\eta_{p, q}(x)\right],
$$

*Bulletin of the Kasan Mathematical Society, vol. 14 (1905), p. 176. 
where $\eta_{p, q}(x)$ approaches zero as $x$ increases, uniformly with respect to $x$ and $q$. The terms of the sequence (1) are, after a certain $p$, less than unity in modulus, since they are roots of functions which approach zero. It follows from (3) that the difference between the $(p+q)$ th and the $p$ th terms of the sequence (1) approaches zero as $p$ increases, uniformly with respect to $x$ and $q$. This proves that the functions of the sequence (1) converge uniformly to an analytic function $\phi(x)$. Furthermore, since

$$
\left\{f_{p}[f(x)]\right\}^{1 / m^{p}}=\left\{\left[f_{p+1}(x)\right]^{1 / m^{p+1}}\right\}^{m},
$$

we find, on allowing $p$ to approach infinity,

$$
\phi f(x)=[\phi(x)]^{m} .
$$

As to the case where $a_{m}$ is not unity, let

$$
F(x)=a_{m}^{1 /(m-1)} f\left(x / a_{m}^{1 /(m-1)}\right) .
$$

The coefficient of $x^{m}$ in $F(x)$ is unity. Let

Since

$$
\Phi(x)=\lim _{p \rightarrow \infty}\left[F_{p}(x)\right]^{1 / m^{p}} .
$$

$$
\left[f_{p}(x)\right]^{1 / m^{p}}=a_{m}^{-1 / m^{p}(m-1)}\left[F_{p}\left(a_{m}^{1 /(m-1)} x\right)\right]^{1 / m^{p}},
$$

it is clear that the functions of the sequence (1) approach as a limit

$$
\phi(x)=\Phi\left(a_{m}^{1 /(m-1)} x\right),
$$

and that $\phi(x)$ satisfies equation (2). This concludes the proof of Boettcher's theorem.

If $\phi(x)$ satisfies the functional equation (2), the function $\epsilon[\phi(x)]^{i}$, where $i$ is any integer, positive or negative, and $\epsilon$ any $(m-1)$ th root of unity, also satisfies the equation. Furthermore, this is the most general solution of equation (2) which is meromorphic in the neighborhood of the origin, for $f(x)$ being given, and the degree of the first term in the development of $\phi(x)$ having been assigned, equation (2) determines the coefficient of the first term of $\phi(x)$ as one of the $(m-1)$ th roots of $a_{m}$, and determines the other coefficients uniquely.

If $f(x)$ is an analytic function with a pole of order $m$ at infinity, we can transform it into a function with a zero of order $m$ at the origin by means of the function $1 / x$. Simultaneously, the functions of the sequence (1), calculated for $f(x)$, will be transformed into the corresponding functions calculated for $1 / f(1 / x)$. Hence the functions of (1) approach a function $\phi(x)$ with a simple pole at infinity, which satisfies equation (2). This fact will be used in $\S \mathrm{IV}$. 


\section{The associated points}

Let $f(x)$ be a rational function of degree greater than unity.

If $f(x)$ is a polynomial, the point infinity has no associates other than itself. If $f(x)$ is a positive or negative power of $x$, the points zero and infinity each have no associates different from themselves. If $f(x)$ can be transformed into a polynomial, or into a power of $x$, by means of a linear function, integral or fractional, there are one or two points, respectively, which have no associates different from themselves.

We shall show that except in the cases described above, every point has an infinite number of associates.

Suppose that a point $c$ has only a finite number of associates. Let $f_{v}(c)$ be one of the consequents which $c$ and all of its associates have in common. Then $f_{\nu}(c)$ can have no associates other than itself, for the $\nu$ th antecedents of such associates would be associates of $c$, and would be distinct from the $\nu$ th antecedents of $f_{\nu}(c)$.

Consider first the case where $f_{\nu}(c)$ is not a fixed point of $f(x)$. The points $f_{v}(c)$ and $f_{v+1}(c)$ have no associates different from themselves. Take a linear function $\phi(x)$, such that $\phi^{-1} f_{\nu}(c)=0$, and $\phi^{-1} f_{\nu+1}(c)=\infty$. Then, relative to the rational function $\phi^{-1} f \phi(x)$, the points zero and infinity, and their consequents, have no associates different from themselves. We must consequently have

$$
\phi^{-1} f \phi(x)=\frac{a+b x^{n}}{x^{n}},
$$

where $n>1$. We have $\phi^{-1} f \phi(\infty)=b$. If $b$ were not zero, it would have associates different from itself, which is impossible. Consequently $f(x)$ can be transformed into the form $a / x^{n}$, and hence also into $1 / x^{n} .^{*}$

Consider now the case where $f_{\nu}(c)$ is a fixed point of $f(x)$. Then $f_{\nu}(c)$ can have no antecedent different from itself, for such antecedents would also be its associates. Let $f(x)$ be transformed with a linear function $\phi(x)$ such that $\phi^{-1} f_{\nu}(c)=\infty$. Then, relative to $\phi^{-1} f \phi(x)$, the point infinity has no antecedent different from itself. The function $\phi^{-1} f \phi(x)$ is consequently a rational integral function.

If there is a point $d$, distinct from $f_{\nu}(c)$, which has only a finite number of associates relative to $f(x)$, a common consequent $f_{\mu}(d)$ of $d$ and its associates must be a fixed point of $f(x)$, for it is easily seen that a function of degree higher than unity cannot be transformed both into a rational integral function and into a negative power of $x$. If $\phi^{-1} f_{\mu}(d)=0$, and $\phi^{-1} f_{\nu}(c)=\infty$, the function $\phi^{-1} f \phi(x)$ will be of the form $c x^{n}$. Hence $f(x)$ can be transformed into the form $x^{n}$.

* The function $a / x^{n}$ is transformed into $1 / x^{n}$ by means of $\phi(x)=a^{1 / n+1} / x$. 
The question as to the number of associates of a point being thus settled, we consider a point $c$, which has an infinite number of associates relative to the rational function $f(x)$. The following facts will be proved:

I. The set of limit points of the associates of $c$ contains a perfect set.

II. The associates of $c$ cannot accumulate about a point of attraction.

III. The consequents of a limit point of the associates of $c$ cannot approach a point of attraction at which the derivative of $f(x)$ is not zero, even should the consequents of $c$ approach the point of attraction.

IV. If the consequents of $c$ tend toward a point of attraction at which the derivative of $f(x)$ is not zero, the associates of $c$ form an isolated set.

V. If $c$ lies within a sufficiently small neighborhood of a point of attraction at which the derivative of $f(x)$ is zero, there exists a simple closed analytic curve, passing through $c$, and around the point of attraction, on which the associates of $c$ are everywhere dense. If $f(x)$ has a pole of order greater than one at infinity, the associates of a point in the neighborhood of infinity lie everywhere dense on a simple closed analytic curve which passes through the point.

We shall prove the result $\mathrm{V}$ first. Let the point of attraction be taken as the point $x=0$. This can always be brought about by transforming $f(x)$ with a function of the form $x+h$. Let the first term in the development of $f(x)$ about $x=0$ be of degree $m$, where $m>1$. By Boettcher's Theorem there exists an analytic $\phi(x)$, with a simple zero at $x=0$, such that

$$
f(x)=\phi^{-1}\left\{[\phi(x)]^{m}\right\} .
$$

The associates of any point, relative to the function $x^{m}$, lie everywhere dense on a circle passing through the point, with center at the origin. If $d$ is an associate of $c$, relative to $f(x), \phi(d)$ will be an associate of $\phi(c)$, relative to the function $x^{m}$. For sufficiently small values of $c$, the function $\phi^{-1}(x)$ transforms the circle passing through $\phi(c)$ and with center at the origin, into a simple closed analytic curve, passing through $c$ and around the origin. The associates of $c$, relative to $f(x)$, are everywhere dense on this curve. This proves the first statement in V. The second statement is proved by transforming $f(x)$ with the function $1 / x$.

In the first case of $\mathrm{V}$, if the degree of $f(x)$ is greater than $m$, the order of the zero of $f(x)$ at $x=0, c$ will have associates which are not included among the associates which were shown above to lie on a curve about the origin. If $c$ is sufficiently small, none of these additional associates can lie within the closed curve, else the point $\phi(c)$ would have an associate relative to the function $x^{m}$ lying within the circle described above. It follows that the associates of a point cannot accumulate about a point of attraction at which the derivative of $f(x)$ is zero. We shall show that the same holds if the derivative of $f(x)$ is not zero, thus completing the proof of II. Let $c$ and $d$ be two points very 
close to the point of attraction which have a common consequent. The consequents of $c$ and $d$ must approach the point of attraction. Let $\nu$ be the smallest integer such that $f_{\nu}(c)=f_{\nu}(d)$. Then the points $f_{\nu-1}(c)$ and $f_{v-1}(d)$, which are very close to the origin, are distinct, and the function $f(x)$ has the same value at both of these points. This is impossible, since the derivative of $f(x)$ does not vanish at the point of attraction. The proof of II is completed.*

Result III follows immediately from the argument given above, for if the consequents of a limit point of the associates of $c$ should tend toward a point of attraction at which the derivative of $f(x)$ is not zero, there would be distinct points in every neighborhood of this point of attraction which have a common consequent. Result IV is a corollary of III.

We shall now prove I. If $d$ is a limit point of the associates of $c$, all of the associates of $d$ are likewise limit points of the associates of $c$. Such a point $d$ cannot be an exceptional point which has only a finite number of associates, since those special points are points of attraction. Thus $D_{1}$, the first derived assemblage of the limit points of the associates of $c$ contains an infinite number of points. Hence the first derived assemblage $D_{2}$, of $D_{1}$, contains at least one point. It is seen readily that if a point belongs to $D_{2}$, all of its associates also belong to $D_{2}$. Hence $D_{2}$ contains an infinite number of points. We see that the derived assemblages

$$
D_{1}, D_{2}, \cdots, D_{n}, \cdots,
$$

all contain an infinite number of points. There exists a closed set of points $D_{\omega}$ common to the sets of the above sequence. The associates of every point of $D_{\omega}$ belong to $D_{\omega}$, so that $D_{\omega}$ contains an infinite number of points. Continuing thus, we can demonstrate the existence of derived assemblages of every transfinite order. It follows from a well-known theorem that the set of limit points of $c$ contains a perfect set. $\dagger$

\section{Polynomials with a common iterate}

We shall discuss in this section the circumstances under which two rational integral functions can have an iterate in common. The discussion given does not apply to the case where the functions are linear, which trivial case can be handled by a direct comparison of the iterates. Also, to avoid the introduction of extra details, we shall bar out the case where the functions can be transformed into multiples of powers of $x$, in which case the iterates can also be compared directly.

\footnotetext{
* If, on transforming $f(x)$ so that $\infty$ is carried into a finite point, this finite point is a point of attraction of the transformed function, we may say that $\infty$ is a point of attraction of $f(x)$. In that case the associates of a point cannot accumulate about $\infty$.

$\dagger$ This argument holds even if the set of associated points is not bounded.
} 
Let us suppose then that the two non-linear polynomials, $f(x)$ and $F(x)$, of degrees $n$ and $N$ respectively, are such that

We must have

$$
f_{p}(x)=F_{P}(x) .^{*}
$$

$$
n^{p}=N^{P} .
$$

There exist therefore three integers, $r, s$, and $S$, such that

$$
n=r^{s}, \quad N=r^{s} .
$$

Consider the two Boettcher functions,

$$
\phi(x)=\lim _{i \rightarrow \infty}\left[f_{i}(x)\right]^{1 / n^{i}}, \quad \Phi(x)=\lim _{i \rightarrow \infty}\left[F_{i}(x)\right]^{1 / N^{i}},
$$

which exist in the neighborhood of $\infty$, at which point they have simple poles. Since

$$
f_{i p}(x)=F_{i P}(x)
$$

for every integer $i$, we must have

$$
\Phi(x)=c \phi(x),
$$

where $c$ is a constant of modulus unity. Then

where

$$
f(x)=\phi^{-1}\left\{[\phi(x)]^{n *}\right\}, \quad F(x)=\phi^{-1}\left\{l[\phi(x)]^{s}\right\},
$$

$$
l=c^{r_{-1}} .
$$

The lemma which follows will be important for. our purposes.

Lemma. If the functions

$$
U(x)=\phi^{-1}\left\{h[\phi(x)]^{r^{u}}\right\}, \quad V(x)=\phi^{-1}\left\{k[\phi(x)]^{r^{r}}\right\},
$$

where $u \geqq v$, are rational and integral, the function

$$
U V^{-1}(x)=\phi^{-1}\left\{h k^{1 / r^{v}}[\phi(x)]^{n-v}\right\},
$$

where any of the determinations of $V^{-1}(x)$ is taken, is also rational and integral.

From the expression of $U V^{-1}(x)$ in terms of $\phi(x)$, we see that $C V^{-1}(x)$ is uniform in the neighborhood of infinity. Let its Laurent development be

$$
G(x)+P(1 / x),
$$

where $G(x)$ is a rational integral function, and $P(1 / x)$ a series of negative powers of $x$. We have

$$
U(x)=G[V(x)]+P[1 / V(x)] .
$$

The Laurent development of $P[1 / V(x)]$ for the point infinity can contain only negative powers of $x$, and since the function $U(x)$ has no negative powers

${ }^{*}$ I solved this problem originally for the case of $p=P$. Dr. Gronwall suggested to me the possibility of generalization. 
in its expansion about infinity, $P[1 / V(x)]$, and hence also $P\left[1 / x_{1}\right.$, must be identically zero. Therefore

which proves the lemma.

$$
U V^{-1}(x)=G(x),
$$

Let $t$ be the smallest integer greater than zero such that there exists a rational integral function of the form

$$
g(x)=\phi^{-1}\left\{e[\phi(x)]^{r^{t}}\right\} .
$$

Then $t$ must be a divisor of $s$. For otherwise we would have

$$
s=i t+j,
$$

where $i$ and $j$ are integers and $0<j<t$, and the function $f\left[g_{-i}(x)\right], g_{-i}(x)$ being the inverse of the $i$ th iterate of $g(x)$, would be a rational integral function of the form

Similarly $t$ is a divisor of $S$.

$$
\phi^{-1}\left\{b[\phi(x)]^{n}\right\} .
$$

If a polynomial whose Boettcher function is $\phi(x)$ is transformed with a linear integral function $\omega(x)$, the Boettcher function of the transformed polynomial will be $\phi[\omega(x)]$. We may therefore suppose that the functions $f(x)$ and $F(x)$ have been transformed with a function of the form $x+h$ so that the development about infinity of the Boettcher function $\phi(x)$ contains no constant term.

Putting $q=s / t$, we see from the lemma above that $f(x)$ is a linear function of $g_{q}(x)$. Since this linear function is of the form

$$
\phi^{-1}[h \phi(x)],
$$

it cannot contain a constant term. We have thus

$$
f(x)=\epsilon_{1} g_{q}(x),
$$

where $\epsilon_{1}$ is a constant. Similarly

$$
F(x)=\epsilon_{2} g_{Q}(x),
$$

where $\epsilon_{2}$ is a constant and where $Q=S / t$.

Expressing $f(x)$ and $g_{q}(x)$ in terms of $\phi(x)$, we have

where

$$
\phi^{-1}\left\{[\phi(x)]^{r}\right\}=\epsilon_{1} \phi^{-1}\left\{\gamma[\phi(x)]^{r}\right\},
$$

Hence

Let

$$
\gamma=e^{\left(r^{t} t-1\right) /\left(r^{t}-1\right)} \text {. }
$$

$$
\phi^{-1}(x)=\epsilon_{1} \phi^{-1}(\gamma x) \text {. }
$$

$$
\phi^{-1}(x)=\alpha x+\frac{\alpha_{1}}{x}+\frac{\alpha_{2}}{x^{2}}+\cdots+\frac{\alpha_{n}}{x^{n}}+\cdots .
$$

Since $f(x)$ is not a multiple of a power of $x, \phi^{-1}(x)$ must actually contain 
negative powers. One sees easily from this expression for $\phi^{-1}(x)$ that $\epsilon_{1}=\gamma$ and that $\epsilon_{1}$ is a root of unity. We must have

$$
\phi^{-1}(x)=\alpha x P\left(1 / x^{m}\right),
$$

where $\epsilon_{1}^{m}=1$, and where $P\left(1 / x^{m}\right)$ is a series of powers of $1 / x^{m}$. Hence, also,

$$
\phi(x)=\frac{x}{\alpha} Q\left(1 / x^{m}\right),
$$

where $Q\left(1 / x^{m}\right)$ is a series of negative powers of $x^{m}$.

We see now from the expression for $g(x)$ in terms of $\phi(x)$ that

$$
g(x)=x^{r^{t}} R\left(1 / x^{m}\right),
$$

where $R\left(1 / x^{m}\right)$ is a polynomial in $1 / x^{m}$.

If we express $F(x)$ and $g_{Q}(x)$ in terms of $\phi(x)$, we have

$$
\phi^{-1}\left\{l[\phi(x)]^{r s}=\epsilon_{2} \phi^{-1}\left\{\delta[\phi(x)]^{r s}\right\},\right.
$$

where $\delta$ is easily expressed as a power of $l$. We see as above that

$$
\epsilon_{2}=\frac{\delta}{l}
$$

and that $\epsilon_{2}$ is a root of unity. Also, if the integer $m$ introduced above is taken as large as possible, so that there are actually two terms in $P\left(1 / x^{m}\right)$ whose exponents differ by $m$, we have $\epsilon_{2}^{m}=1$.

We have

$$
g_{q}\left(\epsilon_{1} x\right)=\epsilon_{1}^{r \cdot} g_{q}(x), \quad g_{Q}\left(\epsilon_{2} x\right)=\epsilon_{2}^{r s} g_{Q}(x) .
$$

Consequently,

Therefore

$$
\begin{aligned}
& f_{p}(x)=\epsilon_{1}^{(r p s-1) /\left(\left(r^{q}-1\right)\right.} g_{p q}(x)=\epsilon_{1}^{\left(n^{p}-1\right) /(n-1)} g_{p q}(x), \\
& F_{P}(x)=\epsilon_{2}^{\left(r^{\left.P S_{-1}\right) /\left(r^{S}-1\right)}\right.} g_{P Q}(x)=\epsilon_{2}^{\left(N^{P}-1\right) /(N-1)} g_{P Q}(x) \text {. }
\end{aligned}
$$

$$
\epsilon_{1}^{(n p-1) /(n-1)}=\epsilon_{2}^{\left(N^{P}-1\right) /(N-1)} .
$$

The conditions on $f(x)$ and $F(x)$ found thus far are easily seen to be sufficient. Our result may be stated as follows: There exists a polynomial $g(x)$, in which the exponents form an arithmetic progression with difference $m$, such that $f(x)$ and $F(x)$ can be derived by transforming

$$
\epsilon_{1} g_{q}(x), \quad \epsilon_{2} g_{Q}(x)
$$

respectively, with a function of the form $x+h$. The constants $\epsilon_{1}$ and $\epsilon_{2}$ satisfy the relations

$$
\begin{gathered}
\epsilon_{1}^{m}=1, \quad \epsilon_{2}^{m}=1, \\
\epsilon_{1}^{\left(n^{p}-1\right) /(n-1)}=\epsilon_{2}^{\left(N^{P}-1\right) /\left(N^{N}-1\right)} *
\end{gathered}
$$

* It should be remembered that $n^{p}=N^{P}$.

Colombia University 